\title{
Estrategias para la integración de Sistemas de Gestión de Calidad y Sistemas de Gestión Documental, en una institución de educación superior ${ }^{*}$
}

Strategies for the integration of quality management systems and document management systems, in a Higher Education Institution

\section{Estratégias para a integração de sistemas de gestão da qualidade e sistemas de gestão documental em uma Instituição de Ensino Superior}

Recibido: 11 de diciembre de 2017

Revisado: 15 de enero de 2018

Aceptado: 26 de marzo de 2018

Ingrid Carolina Moreno Rodrígue: ${ }^{* *}$

Universidad de La Salle

\footnotetext{
Artículo de resultado de investigación. DOI: http://dx.doi.org/10.15332/s2145-1389.2018.0001.06

** Magíster en Gestión Documental y Administración de Archivos. Especialista en Administración y Gerencia de Sistemas de Gestión de Calidad. Administradora de Empresas. Línea de investigación en Gestión, Entorno y Competitividad de las Organizaciones, Universidad de La Salle. Correo electrónico: ingridcmoreno@usantotomas.edu.co y cmrcalidad21@gmail.com
} 


\section{RESUMEN}

Este artículo presenta los resultados de una investigación orientada a identificar la integración y trabajo colaborativo que puede existir desde las dos áreas que gestionan información en una IES, Sistema de Gestión de Calidad (ISO 9001) y Gestión Documental, facilitando la integración de metodologías, el cumplimiento de la normativa tanto interna como externa, optimización de recursos y consolidación de planes de mejoramiento. Se estudian los antecedentes que permiten contextualizar la investigación e identificar los ámbitos en los cuales se han presentado propuestas de integración de estos dos modelos de gestión. Posteriormente, a través de la revisión teórica, se abordan los ejes más representativos, encontrándose que la composición de estrategias se da a partir de concebir planes (objetivos), metas y políticas (categorías). Una vez definida la metodología de estudio a través de un enfoque cualitativo, se aplica como instrumento de recolección de información las entrevistas, seleccionando un grupo de individuos que hacen parte del Sistema de Gestión de Calidad de la institución y expertos en gestión documental, se analizan los resultados obtenidos en la aplicación del instrumento desde el enfoque de las mencionadas categorías, para finalmente formular las estrategias de integración.

Palabras clave: estrategia, integración, sistemas de gestión, calidad, gestión documental.

\section{ABSTRACT}

This article presents the results of a research oriented to identify the integration and collaborative work that can exist from the two areas that manage information in a Higher Education Institution, Quality Management System (ISO 9001) and Document Management, facilitating the integration of methodologies, the compliance with internal and external regulations, resource optimization and consolidation improvement plans. The backgrounds are studied to contextualize the research and identify the areas in which proposals for the integration of these two management models have been presented. Subsequently, through the theoretical review, the most representative themes are addressed, finding that the composition of strategies is given from conceiving plans (objectives), goals and policies (categories). Once the study methodology has been defined, a qualitative approach is applied as an instrument for collecting information, interviews, selecting a group of individuals that are part of the institution's quality management system and experts in document management, analyzing the results obtained in the application of the instrument from the approach of the mentioned categories, to finally formulate the integration strategies.

Keywords: Strategy, integration, management systems, quality, document management.

\section{RESUMO}

Este artigo apresenta os resultados de uma investigação orientada para identificar a integração e trabalho colaborativo que pode existir entre as duas áreas que gerenciam informações em uma IES, Sistema de Gestão da Qualidade (ISO 9001) e gestão documental, facilitando a integração de metodologias ao cumprimento da normativa interna e externa, optimização de recursos e a consolidação de planos de melhoria. Estudam-se os antecedentes que permitem contextualizar a investigação nos quais foram apresentadas propostas de integração desses dois modelos de gestão. Posteriormente, através da revisão teórica, são abordados os eixos mais representativos, constatando que a composição das estratégias é dada a partir de conceber planos (objetivos), metas e políticas (categorias). 
Uma vez definida a metodologia de estudo, enfoque qualitativo, aplica-se como instrumento de coleta de informação entrevistas, selecionando um grupo de indivíduos que fazem parte do sistema de gestão da qualidade da instituição e especialistas em gestão documental, analisando os resultados obtidos em a aplicação do instrumento desde 0 enfoque das categorias mencionadas, para finalmente formular as estratégias de integração.

Palavras-chave: estratégia, integração, sistemas de gestão, qualidade, gestão documental.

\section{INTRODUCCIÓN}

El desarrollo de la investigación centra su interés en generar estrategias que permitan la integración de los sistemas de gestión como herramienta facilitadora de la calidad de las organizaciones, del control y administración de los documentos concebidos en su quehacer diario. Aspectos necesarios para la exitosa gestión empresarial, que se deriva de la toma ágil y consiente de decisiones acertadas y fundamentadas.

Se plantea como objetivo específico en la investigación realizada, el diseñar estrategias para la integración del Sistema de Gestión de Calidad y el Sistema de Gestión Documental en una institución de educación superior, a partir de tres objetivos específicos: 1) establecer un plan de acción orientado a la integración de las metodologías del sistema de gestión de calidad y la gestión documental, 2) definir las metas que se pretenden alcanzar frente a la integración de los sistemas de gestión analizados, 3) proponer las políticas que guíen la implementación o aplicación práctica de la integración de los sistemas estudiados.

El desarrollo de la investigación se fundamenta a partir de la interpretación dada al concepto "estrategia", en donde algunos autores como Chandler (1962), la explican como la forma amplia que comprende la definición de los objetivos, acciones y recursos que orientan el desarrollo de una organización, incluyendo la determinación de las metas y objetivos a largo plazo, la adopción de cursos de acción y la consecución de los recursos necesarios para el logro de dichas metas, otros como Koontz (1990), definen la estrategia como aquellos patrones de los objetivos, por medio de los cuales se le provee una dirección unificada a la organización.

La importancia de la gestión documental radica en que incluye aspectos relevantes directamente relacionados con el cambio cultural en la organización, asociados a los sistemas de gestión y relacionados directamente con la gestión del conocimiento, entre estos se destacan: plantear que la organización exija a su personal documentarse correctamente, tomar conciencia de la importancia de la documentación y que en los procesos de comunicación interna se haga referencia a la gestión documental (Álvarez, Castro y Peña, 2013).

Contar con sistemas que integran la gestión de los documentos y de la calidad en las instituciones de educación superior, se convierte en una excelente alternativa para modernizar y administrar los archivos como fuente fundamental de información tanto académica como administrativa, valiéndose de las normativas aplicables desde los estándares impartidos por el Archivo General de la Nación y el Estado Colombiano (González, 2007).

La constante disyuntiva que representa para las organizaciones responder a dos frentes que proponen gestionar la información documentada de manera controlada, lógica y coherente, se ha convertido en una problemática, principalmente en las entidades públicas del Estado colombiano. Desde la definición de un Programa de Gestión Documental claro alineado con los procesos de calidad, se puede concebir un instrumento de control y mejora en los procesos archivísticos 
a partir de la creación de los documentos hasta su disposición final (Díaz, 2009).

La implantación de sistemas de gestión de documentos como medio facilitador para el trabajo y desarrollo de las organizaciones modernas que han cambiado la forma en la cual documentan sus procesos, se convierte hoy por hoy en una estrategia diferenciadora, tomando especial valor a partir de entender el enfoque integrador que da el contar con el apoyo de un sistema de gestión de calidad que soporte la gestión de la información documentada generada (Bustelo, 2011).

Es indispensable para la articulación de ambas gestiones (G. Calidad, G. Documental), comenzar con la producción de los lineamientos en calidad y gestión documental, debido a que la información contenida en los instrumentos archivísticos deben ser el reflejo de los realizados para calidad (Puentes, 2018), esto significa determinar de forma conjunta el quehacer en la identificación y gestión de los documentos, desde el control a través de las TRD (Tablas de Retención Documental) y la concepción desde los documentos que conformen el sistema de gestión de calidad, procedimientos, manuales, guías, entre otros.

En los últimos años ha venido en aumento la conglomeración de normas creadas para orientar la gestión de sistemas de calidad y sistemas de gestión documental, la evolución que ha tenido Colombia en temas normativos y reglamentarios, ha propiciado en gran medida dicha conglomeración, sumado a ello se evidencian necesidades surgidas en las instituciones públicas, orientadas a realizar la gestión de sus actividades por medio de metodologías que aseguren el seguimiento, control y mejora continua en todos sus procesos. En estas situaciones se apoya la creación de normas para la implementación de sistemas de gestión de calidad y el modelo estándar de control interno, dinámica reglamentada a partir de la Ley 498 de 1998, por la cual se regula el ejercicio de la función administrativa, determina la estructura y se definen los principios y reglas básicas de la organización y funcionamiento de la administración pública, posteriormente se da la expedición de la Ley 872 de 2003, por medio de la cual se crea el sistema de gestión de la calidad en la Rama Ejecutiva del Poder Público y en otras entidades prestadoras de servicios (Hernández, 2011).

En el ámbito de la calidad para las organizaciones del sector privado se siguen los lineamientos y requisitos establecidos en la NTC ISO 9001, con la aparición del Decreto 2746 el gobierno colombiano reconoce al Instituto Colombiano de Normas Técnicas - Icontec, como el primer ente certificador del país, a partir de su reconocimiento es cuando se da inicio al auge de las certificaciones en las empresas colombianas, mantenimiento y mejora de los sistemas de gestión de calidad.

La evolución de los sistemas de gestión documental, se da en el marco regulatorio de la Ley 80 de 1989, por la cual se crea el Archivo General de la Nación AGN. Para el año 2000 nace la Ley 594, Ley General de Archivos, siendo la norma fundamental que regula la administración de los archivos en Colombia, su ámbito de aplicación abarca toda la administración pública, incluyendo las tres ramas del poder público, las entidades privadas que cumplen funciones públicas y los particulares que poseen archivos de interés cultural (AGN, 2014).

Con la Ley 489 de 1998 se obliga a las entidades del sector público a establecer un sistema interno de gestión documental como parte del sistema de información administrativa del sector público, y años más tarde se complementa con el desarrollo de la Ley 872 de 2003 y el Decreto Reglamentario 4110 de 2004, que obligan a las entidades públicas a implementar sistemas de gestión de la calidad, buscando así involucrar al proceso de gestión documental. Conforme a la Ley 87 de 1993 y su Decreto Reglamentario 1599 de 2005, las entidades públicas deben implementar sistemas de 
control interno por procesos y el componente información a la gestión documental.

De la mano con la normatividad desarrollada para el sector público, nacen estándares internacionales orientados a la implementación de sistemas de gestión documental, control de documentos y registros como la ISO 15489, MOREQ Model Requirements for Management of Electronic Records y la ISO 30300.

Esta proliferación de normativas hace necesaria la generación de estrategias que permitan materializar de forma práctica las metodologías propuestas, establecer un vínculo e interrelación propia de los modelos de sistemas de gestión, y a su vez propiciar escenarios que coadyuven a la toma de decisiones y mejora continua en las organizaciones, hablando un lenguaje común.

Por lo anterior se plantea, ¿̇cuáles son las estrategias para integrar sistemas de gestión documental y sistemas de gestión de calidad, en el marco del logro efectivo de los objetivos organizacionales?

\section{METODOLOGÍA}

La investigación se realiza bajo el enfoque de investigación cualitativa, por medio de la cual se logre estudiar en su contexto las estrategias para la integración del Sistema de Gestión de Calidad y el Sistema de Gestión Documental en la institución objeto de estudio, identificando a través de entrevistas las nociones que sobre estos sistemas tienen quienes conforman una parte de la comunidad universitaria.

El tipo de estudio desarrollado es el estudio cualitativo de caso, centrando la muestra en un caso específico: la oficina encargada del mantenimiento y mejora del Sistema de Gestión de Calidad de la institución. El caso permite estudiar con mayor comprensión y claridad sobre el tema en una población en particular.
Dicho estudio, representa una oportunidad práctica para la integración de Sistemas de Gestión de Calidad y Gestión Documental, ante la gran conglomeración de normas en temas de calidad y gestión de documentos que han venido formalizándose en Colombia; a través de "la recolección, el análisis y la presentación detallada y estructurada de información sobre un grupo de la institución" (Galeano, 2012, p. 68).

Indica el autor que "si bien el estudio de caso puede acudir a variedad de técnicas para recolectar información, las más utilizadas son la observación directa (intensiva, participativa); la entrevista (estructurada, semiestructurada y en profundidad), y la revisión documental" (Galeano, 2012, p. 75).

La propuesta implica conocer el punto de vista de partes internas en la organización ligadas a la gestión de calidad y la gestión documental, y su percepción en el tema, teniendo como base las técnicas de investigación citadas por María Eumelia Galeano, como medios de recolección para el estudio de caso, se define como técnica aplicable:

- Entrevistas semiestructurada: la selección de los participantes se realiza a través de la técnica que Fernández propone, precisa el muestreo no probabilístico por conveniencia, en el cual "se determina la muestra fundamentado en escoger de forma intencional y por su nivel de conocimiento y participación en el tema, a los individuos" (Fernández, 2004, p. 154). Se llevan a cabo entrevistas a funcionarios y directivos, con el fin de identificar la percepción que estos tienen del tema y así recolectar información relevante, realizando preguntas abiertas, dando la oportunidad de recibir diferentes matices de respuesta, permitiendo así entrelazar los temas de interés (Ruiz, 2012).

Fundamentados en el tipo de estudio a desarrollar, se selecciona la población teniendo como criterios dos 
enfoques o elementos (Bonilla, 2005) que permiten la definición de la muestra: la adecuación, significa que se conforma por personas que son representativas para la investigación y su suficiencia, seleccionando individuos que participen activamente en la gestión del sistema de calidad, y por otro, que sean expertos en gestión documental, que por sus conocimientos identifiquen las características al momento de alienar los Sistemas de Gestión de Calidad y Gestión Documental, como mecanismo facilitador y orientador para gestionar la documentación y los procesos de la institución, impactando positivamente en el logro de los objetivos organizacionales previstos.

La investigación se estructura en tres fases que comprenden:

\section{Primera fase}

La primera fase se desarrolla teniendo en cuenta los tópicos orientados a contextualizar el desarrollo de la pregunta de investigación, uno enfocado a la revisión de las investigaciones realizadas en el tema, antecedentes; y segundo al despliegue de los objetivos trazados orientados a: establecer a través de un plan de acción la integración de las metodologías para la gestión de calidad y la gestión de documentos (plan), definir las metas que se pretenden alcanzar con la integración de los dos sistemas propuestos (metas), y finalmente proponer las políticas que guíen la implementación para la integración de los sistemas de gestión analizados (políticas).

\section{Segunda fase}

Una vez definidos los objetivos específicos, se procedió a la construcción y aplicación del instrumento de recolección de información seleccionado: la entrevista semiestructurada. Se buscó identificar las orientaciones de los entrevistados en relación con el plan de acción, las metas y políticas que pueden plantearse para la integración de los dos sistemas foco de la presente investigación.

El instrumento se fundamenta en una serie de preguntas orientadas al grupo de seleccionados en la población y muestra requerida, al ser una entrevista semiestructurada, da pie al diálogo abierto y al intercambio de posiciones, por lo cual se constituye en una muy buena herramienta que permite al entrevistado y entrevistador establecer una conversación amena y de doble vía.

Una vez diseñado el instrumento, se aplicó una prueba piloto que permitió realizar los ajustes y mejoras necesarias para finalmente, según la población escogida, aplicar el instrumento definitivo.

\section{Tercera fase}

Luego de realizadas las entrevistas como fuente de información primaria, se procede a realizar el respectivo análisis de los resultados obtenidos, a través de la metodología establecida por Bonilla (2005), por medio de la cual se identifican las temáticas que enmarcan la investigación, a partir de las cuales se asocian las categorías y la información recolectada.

Teniendo como base la información obtenida en el proceso de entrevistas, se realiza la categorización y codificación respectiva, tomando los datos que tienen sentido y se pueden correlacionarse con cada categoría. 
Tabla 1. Criterios de categorías y subcategorías

\begin{tabular}{|c|c|}
\hline $\begin{array}{l}\text { CATEGORÍAS } \\
\text { CRITERIOS }\end{array}$ & $\begin{array}{l}\text { SUBCATEGORÍAS } \\
\text { CRITERIOS }\end{array}$ \\
\hline \multirow{2}{*}{$\begin{array}{l}\text { Plan } \\
\text { Según Serna (2012), los planes de acción son las tareas que deben realizar cada unidad } \\
\text { o área, para concretar las estrategias en un plan operativo que permita su monitoria, } \\
\text { seguimiento y evaluación. }\end{array}$} & Análisis del entorno. \\
\hline & $\begin{array}{l}\text { Recursos disponibles (físicos, económicos, } \\
\text { humanos, temporales). }\end{array}$ \\
\hline $\begin{array}{l}\text { Metas } \\
\text { La meta está directamente relacionada con las definiciones existentes frente al concepto } \\
\text { estrategia, algunas definiciones de autores como Alfred (1962), la definen como la forma } \\
\text { amplia que comprende la definición de los objetivos, acciones y recursos que orientan el } \\
\text { desarrollo de una organización. }\end{array}$ & Metas a corto, mediano y largo plazo. \\
\hline \multirow{5}{*}{$\begin{array}{l}\text { Políticas } \\
\text { Carlota Bustelo (2011), desde la mirada de los sistemas de gestión, los define como el } \\
\text { conjunto de elementos interrelacionados o que interactúan en una organización con el fin de } \\
\text { establecer políticas y objetivos, y los procesos para alcanzarlos, identifica las políticas como } \\
\text { las directrices a implementar en la gestión de los sistemas, puntualmente en los archivos. }\end{array}$} & Asignación y manejo de recursos. \\
\hline & Planificación. \\
\hline & Organización. \\
\hline & Ejecución. \\
\hline & Control. \\
\hline
\end{tabular}

Fuente: elaboración propia.

\section{RESULTADOS Y DISCUSIÓN}

\section{Estrategias para la integración}

Se plantean como estrategias de integración, asociadas

a los beneficios identificados las siguientes:

Tabla 2. Estrategias generales

\begin{tabular}{|c|c|}
\hline ESTRATEGIAS & BENEFICIOS \\
\hline $\begin{array}{l}\text { 1. Compromiso de la dirección: establecer por medio de una } \\
\text { directriz institucional el compromiso de la alta dirección con } \\
\text { la cultura archivística y de calidad en la institución: política } \\
\text { integrada. }\end{array}$ & $\begin{array}{l}\text { - Compromiso institucional. } \\
\text { - Contar con los recursos requeridos. } \\
\text { - Hacer parte de la cultura organizacional. } \\
\text { - Liderazgo. } \\
\text { - Participación en la generación de los documentos, desde la creación } \\
\text { de procesos o ajustes en la estructura orgánica funcional de la } \\
\text { institución. } \\
\text { - Optimización de recursos. } \\
\text { - Planificación. } \\
\text { - Gestión integradora de procesos. }\end{array}$ \\
\hline $\begin{array}{l}\text { 2. Identificación de necesidades y alcance: identificar y } \\
\text { documentar sistemáticamente, las necesidades de tipo operativo } \\
\text { y de soporte, para lograr objetivamente el cambio. }\end{array}$ & $\begin{array}{l}\text { - Permite determinar los documentos y actividades que deben ser } \\
\text { documentadas e incorporadas para su control y mejora. } \\
\text { - Dimensiona el alcance documental necesario, proveniente del } \\
\text { análisis del proceso. } \\
\text { - Toma de decisiones basada en hechos puntuales. } \\
\text { - Control de recursos, al generar la documentación valiosa para la } \\
\text { consulta, toma de decisiones y de cumplimiento de ley, no se incurre } \\
\text { en costos innecesarios: Costos de la no calidad. }\end{array}$ \\
\hline
\end{tabular}




\begin{tabular}{|c|c|}
\hline ESTRATEGIAS & BENEFICIOS \\
\hline $\begin{array}{l}\text { 3. Soporte tecnológico: adquirir equipos tecnológicos que faciliten } \\
\text { el levantamiento de la información, fácil control, uso y consulta } \\
\text { de esta. Aprovechamiento de un software especializado } \\
\text { que permita hacer más interactiva la participación de los } \\
\text { funcionarios, con el fin de facilitar la comprensión del enfoque } \\
\text { por procesos y el manejo de la información. }\end{array}$ & $\begin{array}{l}\text { - Capacidad. } \\
\text { - Acceso. } \\
\text { - Consulta. } \\
\text { - Seguridad. } \\
\text { - Organización. } \\
\text { - Interacción. } \\
\text { - Control. } \\
\text { - Aprovechamiento de recursos. }\end{array}$ \\
\hline $\begin{array}{l}\text { 4. Participación activa: establecer metodologías de participación } \\
\text { activa de los funcionarios. Sensibilización y capacitación en } \\
\text { requisitos de ley, normas, usuario y organización. Formación de } \\
\text { auditores internos en las dos normas: ISO } 9001 \text { - ISO } 30301 .\end{array}$ & $\begin{array}{l}\text { - Personal competente. } \\
\text { - Involucramiento de las partes. } \\
\text { - Construcción colectiva. } \\
\text { - Cumplimiento de requisitos. } \\
\text { - Liderazgo. } \\
\text { - Administración y gestión del conocimiento. }\end{array}$ \\
\hline $\begin{array}{l}\text { 6. Documentar lo necesario: identificar los documentos en } \\
\text { cada actividad del proceso, incluyendo la definición de los } \\
\text { responsables de dichos documentos, como parte de la gestión } \\
\text { de la documentación. }\end{array}$ & $\begin{array}{l}\text { - Claridad en las responsabilidades. } \\
\text { - Diseño e implementación de un sistema de gestión de documentos. }\end{array}$ \\
\hline $\begin{array}{l}\text { 7. Comunicación y divulgación: definir y divulgar las políticas } \\
\text { para regular el acceso a los documentos basados en los requisitos } \\
\text { legales y organizacionales. }\end{array}$ & \multirow{2}{*}{$\begin{array}{l}\text { - Asegura la integridad. } \\
\text { - Controla el acceso, recuperación y uso de la información. } \\
\text { - Permite la autenticidad. } \\
\text { - Ayuda en los procesos de conservación. } \\
\text { - Trazabilidad. } \\
\text { - Seguridad de la información. }\end{array}$} \\
\hline $\begin{array}{l}\text { 8. Seguridad de la información: definir e implementar políticas y } \\
\text { procedimientos para garantizar la integridad y seguridad de los } \\
\text { documentos. }\end{array}$ & \\
\hline $\begin{array}{l}\text { 9. Seguimiento y control: realizar ejercicios de control a través de } \\
\text { auditorías internas al sistema de gestión documental de la mano } \\
\text { con las auditorias de gestión de calidad. }\end{array}$ & $\begin{array}{l}\text { - Identificar fortalezas. } \\
\text { - Identificar mejoras. } \\
\text { - Toma de decisiones para la corrección y prevención. }\end{array}$ \\
\hline $\begin{array}{l}\text { 10. Mejoramiento continuo: implementar la norma ISO } 30301 \\
\text { en la institución, de la mano con el Sistema de Gestión de } \\
\text { Calidad, bajo la estructura de alto nivel, que permita la fácil } \\
\text { integración y sinergia de los dos sistemas. }\end{array}$ & $\begin{array}{l}\text { - Metodologías claras para la toma de decisiones. } \\
\text { - Optimización en la gestión de recursos. } \\
\text { - Alineación de objetivos organizacionales vs. metodologías de gestión } \\
\text { y control. } \\
\text { - Importancia a las partes interesadas. } \\
\text { - Análisis integral del contexto organizacional. }\end{array}$ \\
\hline
\end{tabular}

Fuente: elaboración propia.

\section{Plan de acción}

El plan de acción propuesto está conformado por las siguientes actividades cruciales para la implementación exitosa de la propuesta formulada:

1. Identificación de las directrices legales aplicables y exigidas vs. la gestión adelantada por el Archivo Central de la institución.
2. Identificación de puntos comunes para la integración del sistema de gestión de calidad y el sistema de gestión documental.

3. Definición de cronograma de actividades.

4. Definición y construcción de políticas archivísticas: valoración, seguridad de la información, preservación. 
Tabla 3. Formulación de planes de apoyo

\begin{tabular}{|l|l|}
\hline \multicolumn{1}{|c|}{ PLANES DE APOYO } & \multicolumn{1}{c|}{ DESCRIPCIÓN } \\
\hline Diseño y elaboración de materiales para divulgación. & $\begin{array}{l}\text { Utilizar diversos canales de comunicación y generar estrategias de comunicación } \\
\text { interna que permitan llegar a toda la comunidad universitaria implicada de forma } \\
\text { práctica y personal. } \\
\text { Canales como: portal web, intranet, sitio de calidad, visitas en las oficinas. }\end{array}$ \\
\hline Plan de capacitación. & $\begin{array}{l}\text { Diseñar el plan de capacitación de la mano con la oficina de personal, de tal forma } \\
\text { que se lleve a cabo un control de los resultados del plan y se institucionalice en las } \\
\text { actividades de formación del personal de la universidad, evaluando la eficacia de } \\
\text { dichas capacitaciones. }\end{array}$ \\
\hline $\begin{array}{l}\text { Compra de software especializado que permita la } \\
\text { integración de los dos sistemas (Documental-Calidad). }\end{array}$ & $\begin{array}{l}\text { La adquisición de un software especializado permite agilizar los procesos de control } \\
\text { yadministración del archivo, facilitando una labor que hoy por hoy es dispendiosa y } \\
\text { prácticamente manual. }\end{array}$ \\
\hline
\end{tabular}

Fuente: elaboración propia.

5. Formación del personal implicado.

6. Identificación y análisis de riesgos.

Liderar desde el equipo de calidad y de archivo, los ejercicios propios para generar la cultura que se requiere, apoyados en capacitaciones, talleres, seminarios, el sistema de gestión de calidad, la programación de capacitaciones, los ejercicios de auditorías internas como método de evaluación de resultados y seguimiento, para la toma de decisiones en corrección, prevención y mejora.

\section{Metas (objetivos)}

El proceso estratégico es un conjunto integrado por decisiones acerca de la definición de objetivos (metas), la elaboración de las estrategias para alcanzarlos y la elección de los cursos de acción que se seguirán para su debida consecución (Chiavenato, 2010).

Es importante tener en cuenta los siguientes instrumentos para lograr la exitosa integración de los Sistemas de Gestión de Calidad y Gestión Documental:
- Tabla de retención documental: existente y actualizada bajo el enfoque por procesos.

\section{- Cuadro de clasificación documental.}

- Tabla de valoración: debe construirse y generar a partir de la creación de las políticas de valoración de los documentos al interior de la institución.

- Programa de gestión documental: fundamentado en la integración con la tabla de retención documental: existente y su respectiva divulgación.

- Ampliación del alcance del Sistema de Gestión de Calidad: lograr que todas las dependencias que conforman la institución trabajen bajo la dinámica de procesos.

\section{Objetivos}

De la mano con el objetivo estratégico de la organización deben definirse objetivos en torno a los sistemas a integrar: 
- Asegurar el control de la información que se produce como consecuencia de la gestión de los procesos administrativos y académicos.

- Afianzar la normativa interna existente en torno a la gestión archivística, con el objetivo de lograr procesos archivísticos eficaces y eficientes.

- Consolidar la cultura de calidad y archivística, fundamentados en la participación de todos los equipos de trabajo que conforman las dependencias de la institución.

\section{Políticas}

La definición de políticas claras en torno a la integración de la gestión de calidad y gestión documental, permite contar con declaraciones fundamentadas del cumplimiento de requisitos generales, propiamente las normas ISO en las cuales se fundamentan dichos sistemas, plantean la definición de política de calidad y política de registros. La norma ISO 9001 (Icontec, 2015) relaciona la política como el compromiso para dirigir la organización, proporcionando un marco de referencia para la definición y revisión de los objetivos de calidad.

Para el desarrollo de la integración de los dos sistemas investigados, se proponen algunas políticas que pueden contribuir a la implementación exitosa de los dos sistemas de gestión:

\section{Gestión de cambio}

La calidad académica, expresada en el marco Proyecto Educativo exige procesos administrativos y académicos claros, transparentes, efectivos y eficaces, que permitan flexibilidad y adaptabilidad a los rápidos y constantes cambios del día a día; además de generar poder de reacción ante las tendencias que obligan a actualizar permanentemente las metodologías y modelos, con el ánimo de alcanzar un adecuado nivel de competitividad, que lleve a la institución a perdurar en el tiempo. Generar una política orientada a la gestión del cambio, no solo apoya y aporta al proceso de inmediata atención, sino a su vez, a todos los procesos o ejercicios que impacten en la gestión de la institución y que implican cambios en la forma de ejecutar las actividades y tareas.

Dicho cambio implica:

a. Creación de conciencia por parte de los funcionarios que conforman la organización, en función de la importancia de los archivos y su gestión.

b. Involucramiento total de todos quienes hacen parte de las unidades académicas y administrativas de la institución.

c. Aprovechamiento de los recursos con los que cuenta la institución.

d. Apoyo y compromiso de la alta dirección en la gestión del cambio propuesto.

Figura 1. Definición de políticas

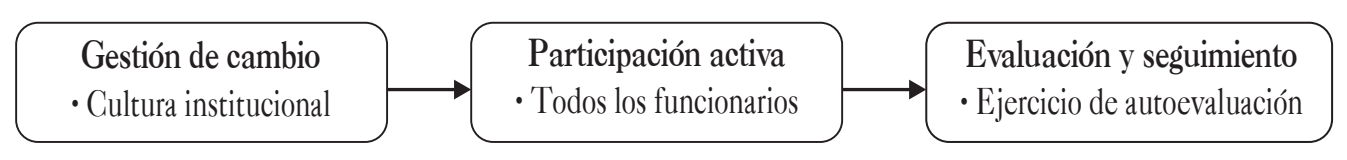

Fuente: elaboración propia 
e. Valorar adecuadamente la importancia de los documentos en la toma de decisiones de la institución y su valor histórico.

\section{Participación activa}

Alineado a los principios de la gestión de calidad, la institución puede generar una política integrada para los sistemas de gestión, orientada a la participación activa de todos los funcionarios implicados, desarrollada a través de:

a. Capacitación de todos los funcionarios.

b. Reconocimiento por la labor emprendida.

c. Escucha activa de ideas y propuestas a todos los niveles.

d. Generar equipos de trabajo.

e. Eliminación de barreras entre dependencias, trabajo bajo el enfoque por procesos.

f. Incentivar un liderazgo positivo y firme.

\section{Seguimiento y medición (indicadores de gestión)}

Los indicadores de gestión como parte central del seguimiento y control cuantitativo de los procesos, permiten identificar el estado actual, evolución y planes de prevención y mejora. Por lo que generar la cultura de la medición en pro de identificar las oportunidades, debilidades y amenazas, debe ser parte del seguimiento periódico a los procesos, definiéndose una política orientada a un proceso de autoevaluación, seguimiento y medición (Sierra, 2012), que aunado a los ejercicios de auditorías, cierren el ciclo de la metodología del PHVA (planear, hacer, verificar y actuar), propuesta por la familia de las ISO 9000.

Se proponen los siguientes indicadores para el seguimiento y control del proyecto formulado:
- Eficacia del plan de trabajo propuesto.

- Eficacia en el cumplimiento del plan de capacitaciones.

- Cumplimiento de los requisitos de calidad en la gestión de los documentos.

- Grado de implementación de la normatividad archivística vigente aplicable.

Al indagar por la planificación, como una de las categorías existentes en la presente investigación, se identifica claramente en la población escogida que existen acercamientos claros a la gestión de calidad como metodología que contribuye al hacer de las actividades en cada unidad, por tanto identifican los planes que son divulgados año tras año, en concordancia con los objetivos o metas que se trazan en el tema, como: ampliación del alcance del sistema, consolidación del equipo de auditores, incremento en el número de procesos, entre otros.

Se identifica a partir de la información recolectada y de los referentes teóricos, que no se han adelantado actividades que permitan dar a conocer a la comunidad académica su aporte e importante participación en la gestión de los documentos y de la información, adicionalmente se están duplicando esfuerzos por controlar los documentos generados como respuesta a la implementación del sistema de gestión de calidad, sin tenerse en cuenta los requisitos propios de la ley general de archivos.

En lo que respecta a las metas, se pudo identificar que existen importantes aportes que los entrevistados hacen en torno a las dos dinámicas investigadas, apoyando su continuidad y mejora, lo cual evidencia que son temas importantes para la institución y aportan directamente a los diferentes proyectos que van surgiendo en todos los niveles de la organización. 
Al hablar de políticas, como tercera categoría, se identifican temas interesantes que aportan a las estrategias de integración, como parte de la percepción de los individuos entrevistados: generación de buenas prácticas, innovación en los procesos de servicios prestados, reducción de los tiempos de atención, fortalecimiento de la comunicación interna, divulgación, y participación e integración.

Por tanto, es indispensable proyectar en el tiempo cada uno de los proyectos estratégicos, definir los objetivos y las estrategias de cada área funcional dentro de los proyectos, así como definir planes de acción concretos, que permitan la ejecución exitosa de los mismos.

\section{CONCLUSIONES}

La implementación de un Sistema de Gestión Documental como metodología transversal a la organización, a través de la integración con el Sistema de Gestión de Calidad, permite replicar a todas las dependencias de la organización los requisitos legales vigentes, a partir de los cuales se dictan las disposiciones en materia de gestión documental en las entidades no solo del orden nacional, departamental y distrital, sino también aquellas entidades privadas que cumplen funciones públicas, como es el caso de la institución objeto de estudio, además de afianzar la cultura de calidad y de archivística en los funcionarios que la conforman, de generar metodologías para la toma de decisiones alineadas a las políticas institucionales, de optimizar recursos, de reducir costos y de trabajar bajo la estructura de alto nivel, metodología orientadora para la implementación integral de las normas ISO.

Se convierte en una oportunidad de administración estratégica para cualquier tipo de organización, dar cumplimiento a las exigencias que en términos de archivística existe, así como gestionar sus procesos de manera organizada, generando información documentada que esté en línea con las herramientas de control que promulgan los sistemas de gestión documental. Este esfuerzo implica a las organizaciones repensarse desde su estructura, asociar los procesos a la organización documental (series y subseries), controlar la información generada desde el sistema de gestión de calidad, facilitar a quienes conforman la organización las técnicas de control alienadas, para que desde la generación de la información hasta su disposición final, sea coherente la articulación de los dos sistemas.

Tomando como base los resultados obtenidos en las entrevistas, desde el análisis de las categorías, se plantean las estrategias para proporcionar la integración de los dos sistemas de gestión relacionados, dando así respuesta a la pregunta de investigación y al objetivo general, formulándose 9 posibles estrategias encaminadas a: el compromiso de la dirección, la identificación de necesidades y alcance, documentar lo necesario, contar con el soporte tecnológico requerido a nivel de hardware y software, participación activa, comunicación y divulgación, seguridad de la información, seguimiento y control; y finalmente mejora continua. En cada una de las estrategias formuladas se identifican los potenciales beneficios, así mismo se logran establecer planes por medio de los cuales se gestionarían dichas estrategias.

\section{REFERENCIAS}

AGN. (2014). Archivo General de la Nación. Recuperado de http://www.archivogeneral.gov.co/Conozcanos/ historia

Álvarez, M., Castro, M., y Peña, G. (2013). Gestión del conocimiento, una perspectiva desde la gestión de la calidad y la gestión documental. Signos, 5(2), 101-118. 
Bonilla, E. (2005). Más allá del dilema de los métodos: la investigación en Ciencias Sociales. Bogotá: Grupo Editorial Norma.

Bustelo, C. (2011). Serie ISO 30300: Sistema de Gestión para los Documentos. Madrid: SEDIC: Asociación Española de Documentación e Información.

Chandler, A. (1962). Strategy and Structure: Strategy and Structure. Cambrige: Strategy and Structure.

Chiavenato, I. (2010). Innovaciones de la administración, tendencias y estrategias, los nuevos paradigmas. Editorial McGraw-Hill Interamericana Editores.

Díaz, C. (2009). Modelo de certificación de calidad para un programa de gestión documental: entidades puiblicas en Colombia. (Tesis de especialización). Bogotá: Universidad de La Salle.

Fernández, A. (2004). Investigación y técnicas de mercado. ESIC Editorial.

Galeano, M. (2012). Estrategias de investigación social cualitativa, el giro de la mirada, (p. 68). La Carreta Editores.

González, M. (2007). Diseño de un modelo de gestión documental en la Universidad de los Llanos, basado en el Sistema de Gestión de Calidad ISO 9001 y NTCGP 1000. (Tesis de especialización). Bogotá: Universidad de La Salle.
Hernández, K. (2011). Sistema de Gestión de Calidad en instituciones puiblicas de educación superior: estudio comparativo Universidad Nacional de ColombiaUniversidad del Valle. (Tesis de maestría). Palmira: Universidad Nacional de Colombia.

Icontec. (2015). NTC ISO 9001. NTC ISO 9001: Sistemas de Gestión de la Calidad. Requisitos. Bogotá: Icontec.

Koontz, H. (1990). Administración. México: McGraw-Hill.

Puentes, M. (2018). Propuesta metodológica para articular la gestión documental con los requisitos de la Ley General de Archivos y la norma técnica internacional ISO 9001:2015. Signos 9(2).

Ruiz, J. (2012). Metodología de la investigación cualitativa. Bilbao: Publicaciones de la Universidad de Deusto.

Serna, H. (2012). Gerencia estratégica, teoría, metodología, alineamiento, implementación y mapas de gestion. 3R Editores.

Sierra, L. (2012). Cómo medir la eficiencia, eficacia y efectividad en archivos: propuesta de indicadores de gestión. San Bernardo, Chile: IV Convención Internacional de Archivistas. 